\title{
NOTE ON NON-COMMUTATIVE SEMI-LOCAL RINGS
}

\author{
YUKITOSHI HINOHARA
}

Our aim in this note is to generalize some topological results of commutative noetherian rings to non-commutative rings. As a supplemental remark of [2] we prove in $\S 1$ that any right ideal of a complete right semi-local ring is closed, and that

$$
\bigcap_{s=1}^{\infty} M J^{s}=(0)
$$

for any finitely generated right module $M$ over a complete right semi-local ring $\Lambda$ where $J$ is the Jacobson radical of $A$.

In $\S 2$ we are concerned with the flatness of modules. C. Lech gave in [7] an ideal theoretical criterion of the flatness of modules over a commutative ring. We notice that his criterion of the flatness is valid for non-commutative rings.

\section{$\S 1$. Non-commutative semi-local rings}

Definition. Let $A$ be a ring with a unit element 1 and $J$ its Jacobson radical; $A$ is said to be right semi-local if the following conditions are satisfied:

(a) $\bigcap_{s=1}^{\infty} J^{s}=0$,

(b) $A$ is right noetherian,

(c) $1 / J$ satisfies the minimum condition on right ideals.

This definition is due to E. H. Batho who studied the basic property of this class of rings in [2].

By virtue of the condition (a), we may introduce a Hausdorff topology (called the $J$-adic topology) in $A$ and construct the completion $\hat{A}$ of $A$ with respect to this topology.

For brevity, we call an ideal $I$ of $A$ a nucleus if $\bigcap_{s=1}^{\infty} I^{s}=0$ and denote the Jacobson radical of the ring $A$ by $J(A)$.

Lemma 1. Let $A$ be a right semi-local ring and $\hat{A}$ the completion of $A$ with respect to the $J(A)$-adic topology. Then we have

Received May 18, 1960. 


$$
\begin{array}{ll}
J(\hat{\Lambda})^{s}=J(A)^{s} \hat{\Lambda}, & \bigcap_{s=1}^{\infty} J(\hat{\Lambda})^{s}=0, \\
J(\hat{\Lambda})^{s} \cap A=J(\Lambda)^{s}, & \Lambda / J(\Lambda)^{s} \cong \hat{\Lambda} / J(\hat{\Lambda})^{s}
\end{array}
$$

for any positive integer $s$.

For the proof we refer to [Theorem 2.3 of 2] and [Theorem 2 of 3].

Lemma 2. Let $A$ be a ring with a unit element 1 . Then we have the relation $N J(A) \mp N$ for any finitely generated right A-module $N(\neq 0)$.

This is [Proposition 2 of 8, p. 200].

Lemma 3. Let $A$ be a ring and $J$ its Jacobson radical. Assume the following conditions for $A$ :

(a) $\Lambda / J$ satisfies the minimum condition on right ideals,

(b) $J$ is a nucleus and has a finite number of right A-basis,

(c) $A$ is complete with respect to the J-adic topology.

Then we have the relation

$$
\bigcap_{s=1}^{\infty}\left(M+F J^{s}\right)=M
$$

for any finitely generated A-submodule $M$ of a free right A-module $F$.

We notice that $\bigcap_{s=1}^{\infty} F J^{s}=(0)$ since $F$ is a free $A$-module and $J$ is a nucleus. Therefore we can define a Hausdorff topology in $F$ by taking $F, F J, F J^{2}, \ldots$ to be neighbourhoods of zero. Then the closure $\bar{N}$ of any submodule $N$ of $F$ is equal to $\bigcap_{s=1}^{\infty}\left(N+F J^{s}\right)$.

Before proving Lemma 3 we prove

Lemma 4. If a submodule $M$ of $F$ is finitely generated we have $\bar{M}=M+M J$.

Proof. We consider the residue class module $F / M J$ of which $(\bar{M}+M J) / M J$ is a submodule. Since $\bar{M} J \subseteq M J$, we have $((\bar{M}+M J) / M J) J=(0)$. Let $\bar{m}$ be any element of $\bar{M}$. Then $\bar{m}$ can be written in the following form, for any positive integer $t$,

$$
\bar{m}=\sum_{i=1}^{n} m_{i} \lambda_{i}+j_{t}
$$

where $\left\{m_{1}, \ldots, m_{n}\right\}$ is a $\Lambda$-basis of $M, \lambda_{i} \in \Lambda$ and $j_{t} \in F J^{t}$. Now $m=(\bar{m} \Lambda+M$ $+M J) / M J$ is a finitely generated module and $\mathrm{m} J=0$. Therefore $\mathrm{m}$ is considered 
as a finitely generated $\Lambda / J$-module. Since $\Lambda / J$ satisfies the minimum condition on right ideals, the module $m$ satisfies the minimum condition on submodules. Consider the descending sequence of submodules

$$
m \supseteq \mathfrak{m} \cap(F J+M J) / M J \supseteq \mathbb{m} \cap\left(F J^{2}+M J\right) / M J \supseteq \cdots,
$$

and there exists an integer $u$ such that

$$
\mathrm{m} \cap\left(F J^{u}+M J\right) / M J=\mathrm{m} \cap\left(F J^{u+1}+M J\right) / M J=\cdots \cdot
$$

The fact that $M J$ is closed implies

$$
\mathrm{m} \cap\left(F J^{u}+M J\right) / M J=(0) .
$$

Let $\bar{m}=\sum_{i} m_{i} \lambda_{i}^{\prime}+j_{u}, j_{u} \in F J^{u}$. Then we have

$$
j_{u}=\bar{m}-\sum_{i} m_{i} \lambda_{i}^{\prime} \in F J^{u} \cap(\bar{m} \Lambda+M) .
$$

Therefore $j_{1 b} \in M J$, i.e. $\bar{m} \in M+M J$. Thus we have $\bar{M} \subseteq M+M J$. The converse inclusion is obvious and we completes the proof of Lemma 4.

Proof of Lemma 3. Since $M$ is a finitely generated module and the twosided ideal $J$ is finitely generated as a right ideal, $M J^{s}$ is finitely generated for any positive integer $s$. Thus we have

$$
M J^{i}=M J^{i}+M J^{i+1} .
$$

Now we are in a position to prove $\bar{M}=M$. Let $\bar{m}$ be any element of $\bar{M}$. Then we have, by virtue of the above relation of submodules,

$$
\begin{aligned}
& \bar{m}=\sum m_{i} \lambda_{i}^{(0)}+m^{\prime}, \lambda_{i}^{(0)} \in \Lambda, \overline{m^{\prime} \in M J \subseteq F J,} \\
& m^{\prime}=\sum m_{i} \lambda_{i}^{(1)}+\bar{m}^{\prime \prime}, \lambda_{i}^{(1)} \in J, \bar{m}^{\prime \prime} \in \overline{M J^{2}} \subseteq F J^{2}, \\
& m^{\prime \prime}=\cdots \cdot
\end{aligned}
$$

Let $\bar{\lambda}_{i}=\sum_{j=0}^{\infty} \lambda_{i}^{(j)}$. Then we have $\bar{m}=\sum_{i} m_{i} \bar{\lambda}_{i} \in M$. This completes the proof of Lemma 3.

As an immediate consequence of Lemma 1 and Lemma 3, we have

THeOREM 1. Let $A$ be a right semi-local ring and $J$ its Jacobson radical. Then any finitely generated right ideal of the completion $\hat{A}$ of $A$ is closed, and therefore there holds the relation $I \hat{\Lambda} \cap A=I$ where $\bar{I}$ is the closure in $A$ of $a$ right ideal $I$ of $A$.

THEOREM 2. Let $A$ be a complete right semi-local ring. Then any right ideal of $A$ is closed. Further, for any finitely generated right $A$-module $M$, we have 
$\bigcap_{s=1}^{\infty} M J^{s}=0$ where $J=J(\Lambda)$.

Proof. There exists an exact sequence of right 1 -modules

$$
0 \rightarrow N \rightarrow F \rightarrow M \rightarrow 0
$$

where $F$ is a finitely generated free $A$-module. Since $A$ is right noetherian, $N$ is finitely generated. Thus we deduce $\bigcap_{s=1}^{\infty}\left(N+F J^{s}\right)=N$, i.e. $\bigcap_{s=1}^{\infty}(F / N) J^{s}=0$. This is the required result since $F / N \cong M$.

By combining this theorem with [Remark 2 of $\S 4$ in 9 ] and [Theorem 3.4 of 2], we have

Corollary. A complete right semi-local ring $A$ is linearly compact as a right A-module in the discrete topology.

Finally we have the following result:

THEOREM 3. Let $A$ be a right noetherian ring with a unit element 1 , and $Q$ a two-sided ideal of $A$ which is a nucleus. If any right ideal $I$ of $A$ is closed with respect to the Q-adic topology, then we have $\bigcap_{s=1}^{\infty} M Q^{s}=(0)$ for any finitely generated right A-module $M$.

Proof. We assume that $\bigcap_{s=1}^{\infty} M Q^{S} \neq 0$ and deduce a contradiction. We consider the set $\widetilde{S}$ of all submodules $S$ such that $\bigcap_{i=1}^{\infty}(M / S) Q^{i} \neq 0$. Let $N^{\prime}$ be a maximal element of $\subseteq$. Then by assumption we have $N=\bigcap_{s=1}^{\infty}\left(N^{\prime}+M Q^{\mathrm{s}}\right) \neq N^{\prime}$. Let $M^{\prime}$ be any submodule of $M$ properly containing $N^{\prime}$. Then $M^{\prime} \supseteq N$ by the maximality of $N^{\prime}$. Let $\bar{m}$ be any element of $M / N^{\prime}$. Then we have $\bar{m} A \cong A / 0(\bar{m})$ where $0(\bar{m})=\{\lambda \in A \mid \bar{m} \lambda=0\}$. Since any submodule of $\bar{m} \Lambda$ contains the unique minimal submodule $N / N^{\prime}$ of $M / N^{\prime}$ (this implies that $M / N^{\prime}$ is sub-directly irreducible), $1 / 0(\bar{m})$ is subdirectly irreducible. Therefore there exists a positive integer $t$ such that $Q^{t} \subseteq 0(\bar{m})$ since $0(\bar{m})$ is closed by assumption. This implies that $\bar{m} Q^{t}=0$. Therefore there exists an integer $s$ such that $\left(M / N^{\prime}\right) Q^{s}=0$ since $M / N^{\prime}$ is finitely generated. This contradicts our assumption $N \neq N^{\prime}$.

\section{$\S 2$. Flatness of modules}

Let $A$ be a ring with a unit element 1 and $M$ a (unitary) left $A$-module. Then the module $M$ is said to be $A$-flat if $\operatorname{Tor}_{n}^{\Lambda}(C, M)=0$ for all right $A$-modules $C$ and all $n>0$. 
Let $\lambda$ be an element of $\Lambda, I$ a right ideal of $\Lambda$ and $M$ a left $\Lambda$-module. Then we use the following notations:

$Z$ for the ring of all integers,

$I M$ for the $Z$-submodule of $M$ generated by the set $I M$,

$(I: \lambda)$ for $\{\mu \in A \mid \lambda \mu \in I\}$ and

$(I M: \lambda)_{M}$ for $\{m \in M \mid \lambda m \in I M\}$.

Theorem 4. Let $A$ be a ring with a unit element 1 . Then, for each left Amodule $M$, the following conditions are equivalent to each other:

(a) $M$ is $A$-flat,

(b) $\operatorname{Tor}_{1}^{\Lambda}(A / I, M)=0$ for each right ideal $I$ of $A$,

(c) i) For any right ideals $I_{1}$ and $I_{2}$ of $\Lambda$, there holds the relation $\left(I_{1} \cap I_{2}\right) M$ $=I_{1} M \cap I_{2} M$, and

ii) For each element $\lambda$ of $A$, there holds the relation $(0: \lambda) M=(0: \lambda)_{M}{ }^{1}{ }^{\prime}$

(d) $(I: \lambda) M=(I M: \lambda)_{M}$ for each right ideal I and each element $\lambda$ of $A$.

The equivalence of the conditions (a) and (b) is an exercise of 4 (see p. 123 of [4]) and the implication $(d) \Rightarrow(a)$ is proved by the same way as in [7], so we prove only the implications $(a) \Rightarrow(c) \Rightarrow(d)$.

To deduce i) of (c) from (a), it suffices to prove

Lemma 5. Let $I_{1}$ and $I_{2}$ be right ideals of $A$ and $M$ a left 1-module. If $\operatorname{Tor}_{1}^{\Lambda}\left(\Lambda / I_{1}+I_{2}, M\right)=0$ we have the relation $\left(I_{1} \cap I_{2}\right) M=I_{1} M \cap I_{2} M^{2}{ }^{2}$

This lemma follows immediately from the exact sequence:

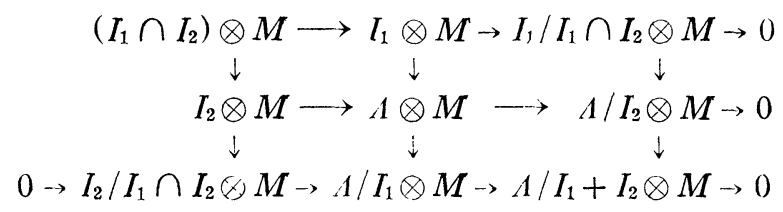

where $\otimes$ means $\otimes_{\Lambda}$.

Proof of the implication (a) $\Rightarrow$ ii) of (c). From the natness of the module $M$, we deduce a commutative exact diagram;

1) A. Hattori called this property of a module torsion-free in [6].

2) The proof of this lemma is a formal generalization of those of [Theorems 5 and 6 in 1 , p. 111]. 


$$
\begin{gathered}
0 \rightarrow(0: \lambda) \otimes M \rightarrow \Lambda \otimes M \rightarrow \Lambda /(0: \lambda) \otimes M \rightarrow 0 \\
\downarrow \\
\downarrow \\
0 \longrightarrow(0: \lambda)_{M} \rightarrow M \longrightarrow \lambda M \rightarrow 0
\end{gathered}
$$

where $\otimes$ means $\otimes_{\Lambda}$. From this diagram we have the required result $(0: \lambda) M$ $=(0: \lambda)_{M}$ by virtue of the well known "five lemma".

Proof of the implication $(\mathrm{c}) \Rightarrow(\mathrm{d})$. It suffices to prove $(I: \lambda) M \supseteq(I M: \lambda)_{\mu}$. Let $m$ be any element of $(I M: \lambda)_{M}$. Then we have $\lambda m \in \lambda(I M: \lambda)_{M}=I M \cap \lambda M$ $=(I \cap \lambda A) M=\lambda(I: \lambda) M$. Therefore there exists an element $m^{\prime} \in(I: \lambda) M$ such that

$$
\lambda m=\lambda m^{\prime}, \quad \text { i.e. } \quad \lambda\left(m-m^{\prime}\right)=0 .
$$

This implies that $m-m^{\prime} \in(0: \lambda)_{M}=(0: \lambda) M \subseteq(I: \lambda) M$. Thus we have $m \in(I: \lambda) M$, and this completes the proof of Theorem 4 .

Remark. As an immediate consequence of Theorem 4, we have the following corollary by combining [Theorem 2 or Corollary of 5]:

A commutative integral domain $A$ is a Prüfer ring if and only if there holds the relation

$$
\left(I_{1} \cap I_{2}\right) I=I_{1} I \cap I_{2} I,
$$

for any ideals $I_{1}, I_{2}$, and $I$ of $A^{3)}$

\section{REFERENCES}

[1] Y. Akizuki and M. Nagata, Modern algebra (in Japanese), Ser. of Modern Math., Kyoritsu-Shuppan Co., Tokyo (1957).

[2] E. H. Batho, Non-commutative semi-local and local rings, Duke Math. J., 24 (1957), pp. $163-172$.

[3] I. S. Cohen, On the structure and ideal theory of complete local rings, Trans. of the Amer. Math. Soc., 59 (1946), pp. 54-106.

[4] H. Cartan and S. Eilenberg, Homological algebra, Princeton Univ., (1956).

[5] A. Hattori, On Prüfer rings, Jour. Math. Soc. Japan, 9 (1957), pp. 381-385.

[6] A. Hattori, A foundation of torsion theory for modules over general rings, Nagoya Math. J., this issue.

[7] C. Lech, Note on multiplicities of ideals, Arkiv för Math., B. 4, H. 1 (1960), pp. 63-86.

[8] N. Jacobson, Structure of rings, Amer. Math. Soc. Colloq. Pub. XXXVII (1956).

[9] D. Zelinsky, Linearly compact modules and rings, Amer. J. of Math., 76 (1953), pp. 79-90.

\section{Tokyo Metroporitan University}

3) This result was suggested to the writer by $T$. Ishikawa. 